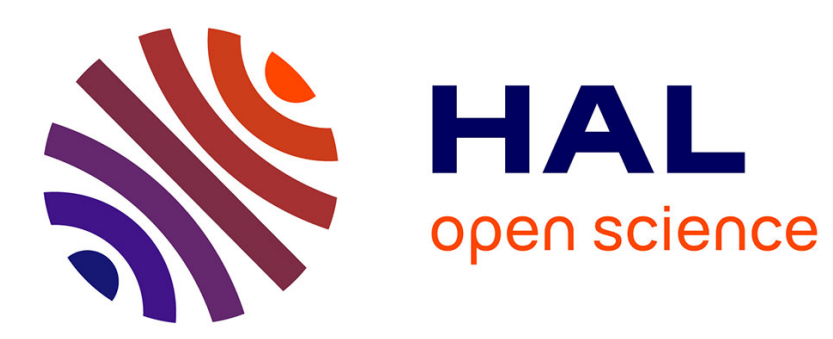

\title{
Two-photoactive-center model applied to photorefractive self-focusing in biased LiNbO3
}

\author{
F. Devaux, J. Safioui, M. Chauvet, R. Passier
}

\section{To cite this version:}

F. Devaux, J. Safioui, M. Chauvet, R. Passier. Two-photoactive-center model applied to photorefractive self-focusing in biased LiNbO3. Physical Review A: Atomic, molecular, and optical physics [1990-2015], 2010, 81 (1), pp.013825. 10.1103/PhysRevA.81.013825 . hal-00476907

\section{HAL Id: hal-00476907 https://hal.science/hal-00476907}

Submitted on 11 May 2021

HAL is a multi-disciplinary open access archive for the deposit and dissemination of scientific research documents, whether they are published or not. The documents may come from teaching and research institutions in France or abroad, or from public or private research centers.
L'archive ouverte pluridisciplinaire HAL, est destinée au dépôt et à la diffusion de documents scientifiques de niveau recherche, publiés ou non, émanant des établissements d'enseignement et de recherche français ou étrangers, des laboratoires publics ou privés. 


\title{
Two-photoactive-center model applied to photorefractive self-focusing in biased $\mathrm{LiNbO}_{3}$
}

\author{
Fabrice Devaux, Jassem Safioui, Mathieu Chauvet, and Rémy Passier \\ Département d'Optique P.M. Duffieux, Institut FEMTO-ST, UMR CNRS 6174, \\ Université de Franche-Comté, F-25030 Besançon Cedex, France
}

(Received 26 August 2009; published 26 January 2010)

\begin{abstract}
We propose a time-dependent, three-dimensional numerical model where iron impurities and polarons are both considered photoactive centers to explain beam self-trapping in biased lithium niobate crystal. It shows that the intensity-dependent behavior reported experimentally is due to the competition between the drift current and the nonlinear photovoltaic current. For low light intensity, beam self-focusing occurs, while beam-splitting is observed at a higher intensity level.
\end{abstract}

DOI: 10.1103/PhysRevA.81.013825

PACS number(s): 42.65.Hw, 42.65.Jx, 42.65.Tg, 72.20.Jv

\section{INTRODUCTION}

As a ferroelectric crystal, lithium niobate $\left(\mathrm{LiNbO}_{3}\right)$ exhibits many properties such as electro-optic, pyroelectric, photovoltaic, or piezoelectric effects. In conjunction with its excellent optical quality, it constitutes a material of choice for optoelectronic devices. In addition, it exhibits photorefractive (PR) properties useful for holographic data storage and in other configurations [1]. The main domain of application of $\mathrm{LiNbO}_{3}$ in optics is in the field of integrated optical circuits. While up to now these circuits have been formed essentially by standard fabrication techniques at the surface of $\mathrm{LiNbO}_{3}$, recent studies have considered it as a host material to form tri-dimensional optical circuits. Either laser scanning [2] or self-trapped beam techniques can be used to induced such guiding structures. In the frame of the latter technique, which is also at the heart of this article, one- (1D) and two-dimensional (2D) PR dark or bright solitons have been demonstrated experimentally in different ferroelectric materials [3-7]. Simultaneously analytical and numerical models have been proposed in order to explain the mechanisms and temporal dynamics of beam self-trapping in PR materials [8-13].

The PR effect is known to be enhanced by doping with transition-metal impurities like iron $(\mathrm{Fe})$, and many PR experiments are consequently performed in $\mathrm{LiNbO}_{3}: \mathrm{Fe}$. In this situation, modeling is adequately performed by the Kukhtarev's one-carrier transport model [14] for low intensity. However, the PR effect in doped and nominally pure $\mathrm{LiNbO}_{3}$ crystal was also investigated at high light intensity with pulsed or focused cw laser beams, but reported effects cannot be described if a single iron photoactive center $\left(\mathrm{Fe}^{2+} \leftrightharpoons \mathrm{Fe}^{3+}+\mathrm{e}^{-}\right)$ is considered. Jermann et al. $[15,16]$ later proposed a model where a second deep center becomes active at high light intensities. This additional center has been identified as $\mathrm{Nb}_{\mathrm{Li}}$ polarons $\left(\mathrm{Nb}^{4+} \leftrightharpoons \mathrm{Nb}^{5+}+\mathrm{e}^{-}\right)$which is an intrinsic defect of the crystal. Quantitative predictions of photorefractive properties in doped and undoped $\mathrm{LiNbO}_{3}$ crystal at low and high light intensities were obtained.

More specifically, PR 2D bright solitons were demonstrated in an unintentionally doped $\mathrm{LiNbO}_{3}$ crystal [7]. In a previous

\footnotetext{
*fabrice.devaux@univ-fcomte.fr; URL: http://www.femto-st.fr/fr/ Departements-de-recherche/OPTIQUE/
}

paper, we proposed a time-dependent, three-dimensional (3D) model with an original approach to numerically solve the light-induced space-charge field [17]. It was later refined to explain the large deflection of self-trapped beams observed in photonic-grade $\mathrm{LiNbO}_{3}$ crystals [18] that is attributed to saturation of the deep ionized donors. Experiments with low power beams are correctly predicted with the above models, but higher beam power reveals a different behavior. The purpose of this article is to describe the physical behavior of beam selftrapping in biased $\mathrm{LiNbO}_{3}$ crystal versus intensity. Both iron impurities and polarons are considered as photoactive centers for charge transport. Note that the studied process opens up the possibility to usefully exploit the PR effect to confine light and in the meantime to avoid the catastrophic optical damage usually observed at high intensity in $\mathrm{LiNbO}_{3}$ crystals. In the first part of the article, a numerical model is presented which reveals the competition between drift and photovoltaic currents as a function of the light intensity. Self-focusing and large bending of a Gaussian beam at low light intensity is still predicted, and additional splitting of the beam in two trapped beams is predicted for higher intensities. Corroborating experimental results are depicted in the second part of the article.

\section{THEORETICAL BACKGROUND AND NUMERICAL MODEL}

From the elaborated two-deep level band transport model where iron ions and polarons are considered as photoactive centers developed by Jermann et al. [15], the following system of equations can be considered:

$$
\begin{aligned}
\frac{\partial N_{\mathrm{Fe}}^{+}}{\partial t}= & \left(s_{\mathrm{Fe}}+s_{\mathrm{FeX}} N_{X}^{+}\right) I\left(N_{\mathrm{Fe}}-N_{\mathrm{Fe}}^{+}\right) \\
& -\left[\left(\gamma_{\mathrm{Fe}} N_{e}+\gamma_{X \mathrm{Xe}}\left(N_{X}-N_{X}^{+}\right)\right] N_{\mathrm{Fe}}^{+},\right. \\
\frac{\partial N_{X}^{+}}{\partial t}= & \left(s_{X} I+\gamma_{X \mathrm{Fe}} N_{\mathrm{Fe}}^{+}\right)\left(N_{X}-N_{X}^{+}\right) \\
& -\left[\left(\gamma_{X} N_{e}+s_{\mathrm{Fe} X} I\left(N_{\mathrm{Fe}}-N_{\mathrm{Fe}}^{+}\right)\right] N_{X}^{+},\right. \\
\frac{\partial N_{e}}{\partial t} & =\frac{\partial N_{\mathrm{Fe}}^{+}}{\partial t}+\frac{\partial N_{X}^{+}}{\partial t}-\frac{1}{e} \frac{\partial \rho}{\partial t},
\end{aligned}
$$




$$
\begin{gathered}
\vec{J}=e \mu N_{e} \vec{E}+\kappa_{\mathrm{Fe}} I\left(N_{\mathrm{Fe}}-N_{\mathrm{Fe}}^{+}\right) \vec{c} \\
+\kappa_{X} I\left(N_{X}-N_{X}^{+}\right) \vec{c}+\mu k_{B} T \vec{\nabla} N_{e}, \\
\frac{\partial \rho}{\partial t}=-\vec{\nabla} \cdot \vec{J}
\end{gathered}
$$

where $N_{e}, N_{\mathrm{Fe}}^{+}$, and $N_{X}^{+}$are, respectively, the densities of free electrons, ionized iron, and ionized polarons. $I$ is the light intensity, $s_{\mathrm{Fe}}, s_{X}$, and $s_{\mathrm{Fe} X}$ are the photoionization coefficents of electron from, respectively, the $\mathrm{Fe}^{2+}$ and $X$ states into the conduction band and from $\mathrm{Fe}^{2+}$ to $X^{+}$states. $\gamma_{\mathrm{Fe}}, \gamma_{X}$, and $\gamma_{X \mathrm{Fe}}$ are the corresponding recombination rates of electrons. While electron recombination from conduction band (CB) to the $X^{+}$state was discarded in Ref. [15], we stress that such an electron transition is considered here and that the $\gamma_{X}$ value is estimated from the assumption that $\gamma_{\mathrm{Fe}} N_{\mathrm{Fe}}^{+} \gg \gamma_{X} N_{X}^{+}$ in $\mathrm{LiNbO}_{3}: \mathrm{Fe}$ [15]. Equation (1d) is the expression of the total current density where drift current, photovoltaic (PV) currents of both photoactive centers, and thermal diffusion are considered. $\kappa_{\mathrm{Fe}}$ and $\kappa_{X}$ are PV coefficients. $\vec{E}$ represents the total electric field in the material. The space-charge density is given by $\rho=e\left(N_{\mathrm{Fe}}^{+}+N_{X}^{+}-N_{A}-N_{e}\right)$, where $N_{A}$ is constant and corresponds to the shallow acceptor density. $e$ and $\mu$ are, respectively, the elementary charge and the mobility of electrons. $k_{B}$ is the Boltzmann constant, and $T$ is the temperature.

In nominally pure $\mathrm{LiNbO}_{3}$ crystal, iron is a deep trap present as an donor impurity with concentration lower than 10 mol parts in $10^{6}$ (ppm) which corresponds approximately to iron density $N_{\mathrm{Fe}}<2 \times 10^{23} \mathrm{~m}^{-3}$. We have previously shown in Ref. [17] that large beam bending can occur due to saturation of ionized donors. It occurs when donor density is of the same order of magnitude as space-charge density $\left(|\rho| / e \sim N_{\mathrm{Fe}}-N_{\mathrm{Fe}}^{+} \sim 2 \times 10^{19} \mathrm{~m}^{-3}\right)$ and consequently when most iron centers are ionized $\left(N_{\mathrm{Fe}}^{+} / N_{\mathrm{Fe}}=0.99\right)$. In addition, the PV effect is known to be more efficient for extraordinary (e) than for ordinary $(o)$ polarized light. In the numerical calculation, PV coefficients for $e$ polarization are assumed to be twice that of PV coefficients for $o$ polarization. Other parameters of the material listed in Table I are taken from [15].

\section{NUMERICAL RESULTS}

First, let us consider the case of an electrically biased crystal, with a static electric field $E_{0}$ applied along the $c$ axis. In addition, the crystal is uniformly illuminated with $e$-polarized light. Because of their energy level position in the band diagram, all polaron centers are supposed to be ionized $\left(N_{X}^{+}=N_{X}\right)$ in the dark. Moreover, most iron centers are supposed to be ionized $\left(N_{\mathrm{Fe}}^{+} / N_{\mathrm{Fe}}=0.99\right)$, and no free electron is present in the dark $\left(N_{e}=0\right)$ because of the presence of shallow acceptors. From these initial conditions, rate equations (1a)-(1c) are numerically solved to give the population densities of the different electric species at steady state. Figure 1(a) shows the typical variation of $N_{\mathrm{Fe}}-N_{\mathrm{Fe}}^{+}$, $N_{X}-N_{X}^{+}$, and $N_{e}$ densities with respect to light intensity for an applied electric field set to $E_{0}=6.5 \times 10^{6} \mathrm{~V} \mathrm{~m}^{-1}$. Then, thanks to Eq. (1d), the component along the $c$ axis of current densities is calculated. Figure 1(b) reveals the competition
TABLE I. $\mathrm{LiNbO}_{3}$ crystal parameters.

\begin{tabular}{lc}
\hline \hline Parameter $(\lambda=532 \mathrm{~nm})$ & Value \\
\hline$n_{o}$ & 2.32 \\
$n_{e}$ & 2.22 \\
$r_{31}\left(\mathrm{~m} \mathrm{~V}^{-1}\right)$ & $9.6 \times 10^{-12}$ \\
$r_{33}\left(\mathrm{~m} \mathrm{~V}^{-1}\right)$ & $32.9 \times 10^{-12}$ \\
$\mu\left(\mathrm{m}^{2} \mathrm{~V}^{-1} \mathrm{~s}^{-1}\right)$ & $7.4 \times 10^{-5}$ \\
$s_{\mathrm{Fe}}\left(\mathrm{m}^{2} \mathrm{~J}^{-1}\right)$ & $10^{-5}$ \\
$s_{X}$ & $5 \times 10^{-5}$ \\
$s_{\mathrm{Fe} X}\left(\mathrm{~m}^{5} \mathrm{~J}^{-1}\right)$ & $3.22 \times 10^{-30}$ \\
$\gamma_{\mathrm{Fe}}\left(\mathrm{m}^{3} \mathrm{~s}^{-1}\right)$ & $1.65 \times 10^{-14}$ \\
$\gamma_{X}{ }^{\mathrm{a}}$ & $<5 \times 10^{-16}$ \\
$\gamma_{X \mathrm{Fe}}{ }^{\mathrm{b}}$ & $10^{-19}$ \\
$\kappa_{\mathrm{Fe}}^{o}\left(\mathrm{~m}^{3} \mathrm{~V}^{-1}\right)$ & $-3.5 \times 10^{-33}$ \\
$\kappa_{\mathrm{Fe}}^{e}$ & $-7 \times 10^{-33}$ \\
$\kappa_{X}^{o}$ & $-21.2 \times 10^{-33}$ \\
$\kappa_{X}^{e}$ & $-42.4 \times 10^{-33}$ \\
$N_{X}{ }^{\mathrm{c}}\left(\mathrm{m}^{-3}\right)$ & $2 \times 10^{26}$ \\
$N_{\mathrm{Fe}}$ & $2 \times 10^{21}$ \\
\hline \hline
\end{tabular}

${ }^{\text {aEstimated value }}$

${ }^{\mathrm{b}}$ Best fit value.

${ }^{\mathrm{c}}$ Estimated values corresponding to $1 \%$ of polarons.

between drift and photovoltaic currents; for low light intensity, the contribution of polarons to PV current is negligible, and consequently, a positive total current given by drift current is present. When intensity increases up to a threshold intensity $I_{\text {th }} \sim 10^{5} \mathrm{~W} \mathrm{~m}^{-2}, \mathrm{PV}$ current due to polarons becomes significant and total PV current compensates drift current. For higher intensities, PV currents become greater than drift current and an inversion of the current sign is observed. Figure 1(c) shows more precisely the evolution of the total current with respect
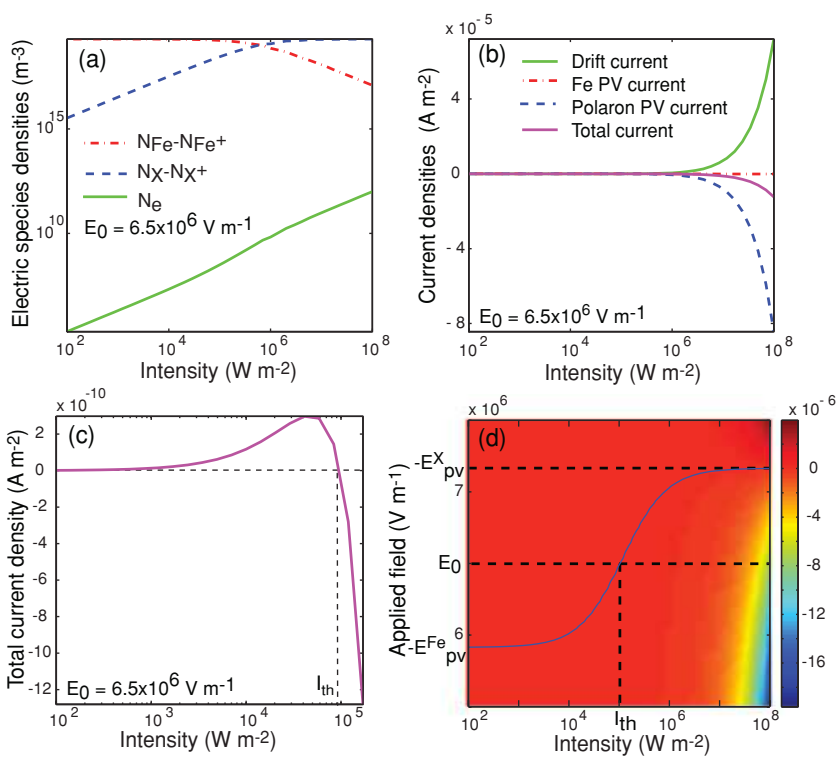

FIG. 1. (Color online) Intensity dependence of population densities at a steady state for polarons, Fe ions, and electrons (a), PV and drift currents densities (b), and total current density (c). Total current density versus light intensity and bias voltage (d) where the solid blue line indicates no net current (exact compensation between $\mathrm{PV}$ and drift currents). 
to the light intensity. The total current first increases with light intensity up to a maximum and then continuously decreases. Since the bias voltage amplitude is an important parameter, Fig. 1(d) gives a picture of the current dependence with respect to light intensity and bias voltage. The blue curve indicates exact compensation between drift and PV currents (no net current). Note that this latter curve also gives the amplitude of the so-called PV field $\left|E_{p v}\right|$ versus light intensity. For low light intensity, the main contribution to photovoltaic current comes from iron centers and PV current increases linearly with intensity which leads to a constant photovoltaic field as for a single center model. The amplitude is $E_{p v}^{\mathrm{Fe}} \sim-6 \times$ $10^{6} \mathrm{~V} \mathrm{~m}^{-1}$ for the considered parameters. For higher intensities, $E_{p v}$ decreases and tends to a new limit value $E_{p v}^{X} \sim-7 \times$ $10^{6} \mathrm{~V} \mathrm{~m}^{-1}$ that corresponds to full ionization of iron centers. Assuming, respectively, $\frac{\partial N_{e}}{\partial t}=0, N_{\mathrm{Fe}}^{+} \simeq N_{\mathrm{Fe}}, N_{X}-N_{X}^{+}=0$ for low intensity and $\frac{\partial N_{e}}{\partial t}=0, N_{X}^{+} \simeq N_{X}, N_{\mathrm{Fe}}-N_{\mathrm{Fe}}^{+}=0$ for high intensity, we can deduce from Eqs. (1a)-(1c), the following expressions of PV fields for $e$-polarized light:

$$
\begin{aligned}
& E_{p v_{e}}^{\mathrm{Fe}}=\frac{\kappa_{\mathrm{Fe}}^{e}\left(\gamma_{\mathrm{Fe}} N_{\mathrm{Fe}}+\gamma_{X} N_{X}\right)}{s_{\mathrm{Fe}} \mu e}=-5.9 \times 10^{6} \mathrm{~V} \mathrm{~m}^{-1}, \\
& E_{p v_{e}}^{X}=\frac{\kappa_{X}^{e}\left(\gamma_{\mathrm{Fe}} N_{\mathrm{Fe}}+\gamma_{X} N_{X}\right)}{s_{X} \mu e}=-7.2 \times 10^{6} \mathrm{~V} \mathrm{~m}^{-1} .
\end{aligned}
$$

Subsequently, $E_{p v}$ versus intensity is perfectly matched by the following equation:

$$
E_{p v}=\frac{E_{p v}^{X}}{1+\frac{I_{c}}{I}}+\frac{E_{p v}^{\mathrm{Fe}}}{1+\frac{I}{I_{c}}}
$$

where $I_{c}=\frac{s_{\mathrm{Fe}} \gamma_{X \mathrm{Fe}} N_{\mathrm{Fe}}}{s_{X}\left(s_{\mathrm{Fe}}+s_{\mathrm{Fe} X} N_{X}\right)}=1.2 \times 10^{5} \mathrm{~W} \mathrm{~m}^{-2}$ corresponds to a critical intensity. $I_{c}$ strongly depends on iron density and is sensitive to wavelength and polarization of light. Moreover, the expression of $I_{c}$ is in agreement with the critical intensity defined in [19] where excitation coefficients $s_{X}$ and $s_{\mathrm{Fe}}$ are assumed to be of the same order of magnitude and where the excitation channel from $\mathrm{Fe}^{2+}$ to $X^{+}$states is discarded. These results are also consistent with the saturation of the refractive index change reported in [15], and Eq. (3) can be used to determine the light intensity threshold responsible for optical damage in $\mathrm{LiNbO}_{3}$ [20]. Finally, to conclude this section, it is important to note that the sign of the total current can be controlled either with light intensity or bias voltage.

Let us now study the case of a nonuniform illumination in a biased crystal. More specifically, propagation of a focused Gaussian beam is considered. From the above development, it is clear that any intensity variation, due for instance to transverse intensity distribution and beam diffraction, induces strong photorefractive current change. From a different point of view, intensity and bias voltage can also be adjusted in order to locally induce a positive or negative current. Consequently, the focusing or defocusing property of the material can be controlled either with beam power or bias voltage; when $E_{0}<-E_{p v}^{\mathrm{Fe}}$ or $E_{0}>-E_{p v}^{X}$, respectively, defocusing or focusing effects dominate whatever beam intensity. For $-E_{p v}^{\mathrm{Fe}}<$ $E_{0}<-E_{p v}^{X}$, beam self-focusing occurs if $I<I_{\text {th }}$, but competition between drift and PV currents is strong when $I>I_{\text {th }}$ and the photorefractive defocusing effect of light could be present along the first diffraction lengths while the focusing effect dominates further away.

In order to expose this behavior, we solve the threedimensional, time-dependent numerical model with the ionized iron ions, polarons, electrons rate equations [Eqs. (1a)(1c)], and the equation that gives the total current [Eq. (1d)]. Starting from initial conditions for population of the different electric species $\left(N_{X}^{+} / N_{X}=1, N_{\mathrm{Fe}}^{+} / N_{\mathrm{Fe}}=0.99, N_{e}=0\right.$, and $\rho=0$ ) and initial light distribution in the volume of the crystal, evolution of the space-charge density $\rho$, and population densities of all the electric species are calculated. By solving Eq. (4) with a three-dimensional discrete Fourier transform, the three components of the space-charge (SC) field $\overrightarrow{E_{\mathrm{sc}}}$ produced by the charge distribution $\rho(\vec{r}) \mathrm{dV}$ in the crystal volume $V$ are deduced ( $d V$ being an elementary volume).

$$
\overrightarrow{E_{\mathrm{sc}}}(\vec{r})=\frac{1}{4 \pi[\varepsilon]} \iiint_{V} \rho\left(\vec{r}^{\prime}\right) \frac{\vec{r}-\vec{r}^{\prime}}{\left|\vec{r}-\vec{r}^{\prime}\right|^{3}} \mathrm{dV} .
$$

Thus, the total electric field in the crystal is given by $\vec{E}=$ $\overrightarrow{E_{0}}+\overrightarrow{E_{\mathrm{sc}}}$. From the calculated electric field, refractive index changes $\Delta n$ induced by Pockels effect is calculated for both $o$ or $e$ polarization $\left(\Delta n_{o}=-\frac{1}{2} n_{o}^{3} r_{13} E_{y}, \Delta n_{e}=-\frac{1}{2} n_{e}^{3} r_{33} E_{y}\right.$, with $E_{y}$ being the component of the total electric field along the $c$ axis). Then, propagation of light in this perturbated medium is performed by a classical split-step Fourier transform method that gives the new light intensity distribution. The whole procedure is repeated until the steady-state regime is reached. The numerical experiment consists in focusing a laser beam at $532 \mathrm{~nm}$ to a waist of $17 \mu \mathrm{m}$ located at the entrance face of a 2-cm-long $\mathrm{LiNbO}_{3}$ crystal which describes closely the experiments reported in the second part of the paper. The laser beam is extraordinary, or the ordinary polarized and beam power is set in the $5-150-\mu \mathrm{W}$ range which corresponds to the $10^{4}-1.7 \times 10^{5} \mathrm{~W} \mathrm{~m}^{-2}$ intensity range including the threshold intensity. In such a case, light-induced hole polarons and light-induced absorption observed for very high intensities $\left(I \gg 10^{6} \mathrm{~W} \mathrm{~m}^{-2}\right)$ are neglected [21]. No background illumination is used.

First we consider the propagation of a low power $9 \mu \mathrm{W}(I=$ $10^{4} \mathrm{~W} \mathrm{~m}^{-2}$ ), $o$-polarized beam with $E_{0}=5 \times 10^{6} \mathrm{~V} \mathrm{~m}^{-1}$ (such as $-E_{p v_{o}}^{X}<E_{0}$ ). In this case, drift current is always greater than PV current which leads to self-focusing of the beam as depicted in Fig. 2. It presents the time dependence of the output beam profile along the $c$ axis along with pictures of the output beam at characteristic times of the process [Figs. 2(b)-2(d)]. As time evolves, self-trapping of the beam is observed and further on beam deflection appears due to saturation of iron centers. Note that such a behavior can also be present when only one deep center is considered [17]. An output-focused beam reaches a width of $10 \mu \mathrm{m}$, and the beam displacement is close to $15 \mu \mathrm{m}$ along the $c$ axis (in the opposite direction to the applied field $E_{0}$ ). For extraordinary polarization, beam self-focusing is also observed, but a stronger Pockels effect leads to overfocusing that finally tends to strongly distort the beam instead of showing beam deflection.

The case of a higher beam power $(140 \mu \mathrm{W}: I=1.5 \times$ $\left.10^{5} \mathrm{~W} \mathrm{~m}^{-2}>I_{\mathrm{th}}\right) e$-polarized focused beam with $E_{0}=6.5 \times$ $10^{6} \mathrm{~V} \mathrm{~m}^{-1}$ (such as $-E_{p v_{e}}^{\mathrm{Fe}}<E_{0}<-E_{p v_{e}}^{X}$ ) is now studied. 


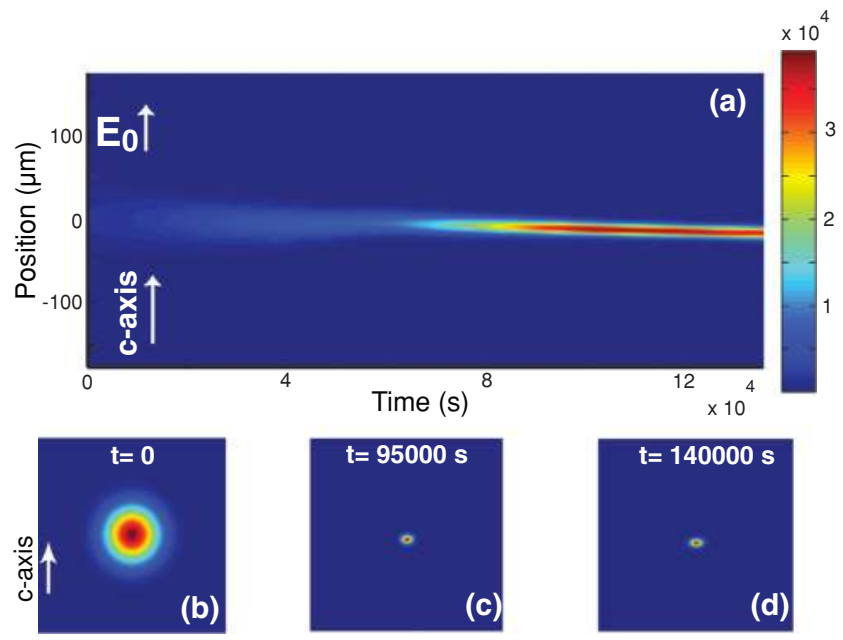

FIG. 2. (Color online) Self-focusing and bending of a low power (input intensity : $I=10^{4} \mathrm{~W} \mathrm{~m}^{-2}<I_{\text {th }}$ ) $o$-polarized Gaussian beam. (a) Output beam profile evolution along the $c$ axis. (b)-(d) Pictures of the output beam at different times.

Figure 3 shows spatial distributions of the PR parameters along the propagation axis and in the transverse plane at the input and output of the crystal at the beginning of the PR process. As input intensity is higher than $I_{\text {th }}$ along the first diffraction lengths [Fig. 3(a)], the total current is negative in the center of the beam while it has a positive value on its edges [Fig. 3(b)]. SC distribution [Fig. 3(c)] induced by such a current gives rise to a positive SC field in the center of the beam and to a negative SC field on both sides of the beam [Fig. 3(d)]. Then by the mean of the Pockels effect, it leads to a defocusing effect in the center of the beam and to a focusing effect on the edges [Fig. 3(e)]. Because of the diffraction, beam intensity decays along propagation $\left(I<I_{\text {th }}\right)$. As a consequence, in the last part of the crystal, the PV current decreases and the total current becomes positive which leads to a focusing effect in the central part of the beam. As the photorefractive effect continues to evolve [Figs. 4(a)-4(e)], competition between defocusing and focusing effects in the initial propagation lengths creates a splitting of the beam in two spots [Fig. 4(a)]. Since the intensity of each spot is smaller than the intensity threshold $I_{\text {th }}$, positive current is still generated in the last part of the crystal [Fig. 4(b)] with transverse spatial distribution similar to the beam intensity profile. With such a particular shape of the total current, new space charges are generated in the center of the beam [Fig. 4(c)]. Figure 4(d) gives a picture of the corresponding electric field. Note that while the initial total electric field in the center of the beam was smaller than $E_{0}$ because of the negative PR SC field [Fig. 3(d)], the SC field induced by central charges now tends to counterbalance the negative SC field induced by surrounding charges. Finally, the corresponding index perturbation exhibits two focusing areas that form two self-trapped beams in the transient regime. If the right parameters are chosen, two PR solitons can be formed. It is important to note that if input beam intensity is larger than twice the intensity threshold splitting, in an even number of spots, greater than two can eventually occur. Figure 5 presents the overall dynamic of this beam-splitting and the self-focusing of the two beams. Two
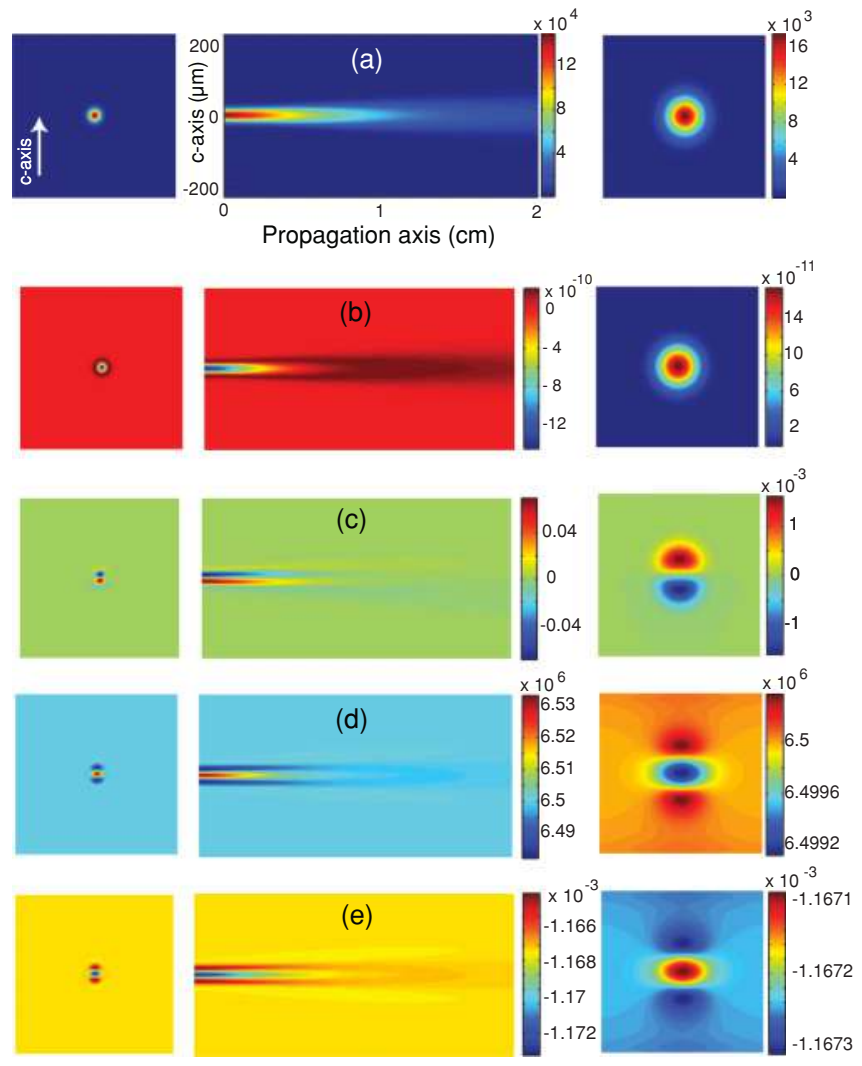

(e)

z

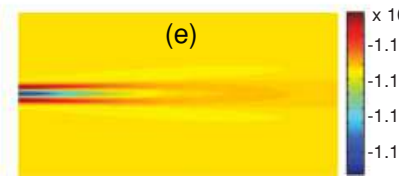

1671

FIG. 3. (Color online) Early stage of the PR process for a $e$-polarized high input power of $140 \mu \mathrm{W}\left(I=1.5 \times 10^{5} \mathrm{~W} \mathrm{~m}^{-2}>\right.$ $I_{\text {th }}$ ) focused beam. Input (left column) and output (right column) transverse spatial distributions and spatial distributions along the $c$ axis versus propagation distance (center column) for (a) beam intensity $\left(\mathrm{W} \mathrm{m}^{-2}\right)$, (b) total current component along the $c$ axis $\left(\mathrm{A} \mathrm{m}^{-2}\right)$, (c) space-charge density $\left(\mathrm{C} \mathrm{m}^{-3}\right)$, (d) total electric-field component along the $c$ axis $\left(\mathrm{V} \mathrm{m}^{-1}\right)$, and (e) extraordinary index modulation.

solitons can be observed but with slightly dissimilar intensity and size [Fig. 5(e)]. Additionally, the trajectories of solitons are not exactly symmetric [Fig. 5(a)]. These phenomena are clear manifestations of a saturation of the iron center.

We emphasize that splitting behavior is also observed with $o$-polarized light. Moreover, as the sign of the total current can be controlled either with light intensity or bias voltage, splitting of the beam in a pair of solitons can be also controlled with bias voltage.

\section{EXPERIMENTAL RESULTS}

At a low power beam, experimental self-focusing and self-bending of PR solitons have already been reported in biased $\mathrm{LiNbO}_{3}$ [7,18]. Therefore, in this last part of the article, we mainly present experimental results confirming our numerical prediction of focusing or splitting of the beam with respect to the beam intensity. Similar to the numerical calculation described in Sec. III, a laser beam at $532 \mathrm{~nm}$ is focused to a waist of $10 \mu \mathrm{m}$ located at the entrance face of a 2-cm-long congruent $\mathrm{LiNbO}_{3}$ crystal as depicted by the input and output pictures of the beam given in Figs. 6(a) and 6(b), respectively. First, beam power is set to a given value 

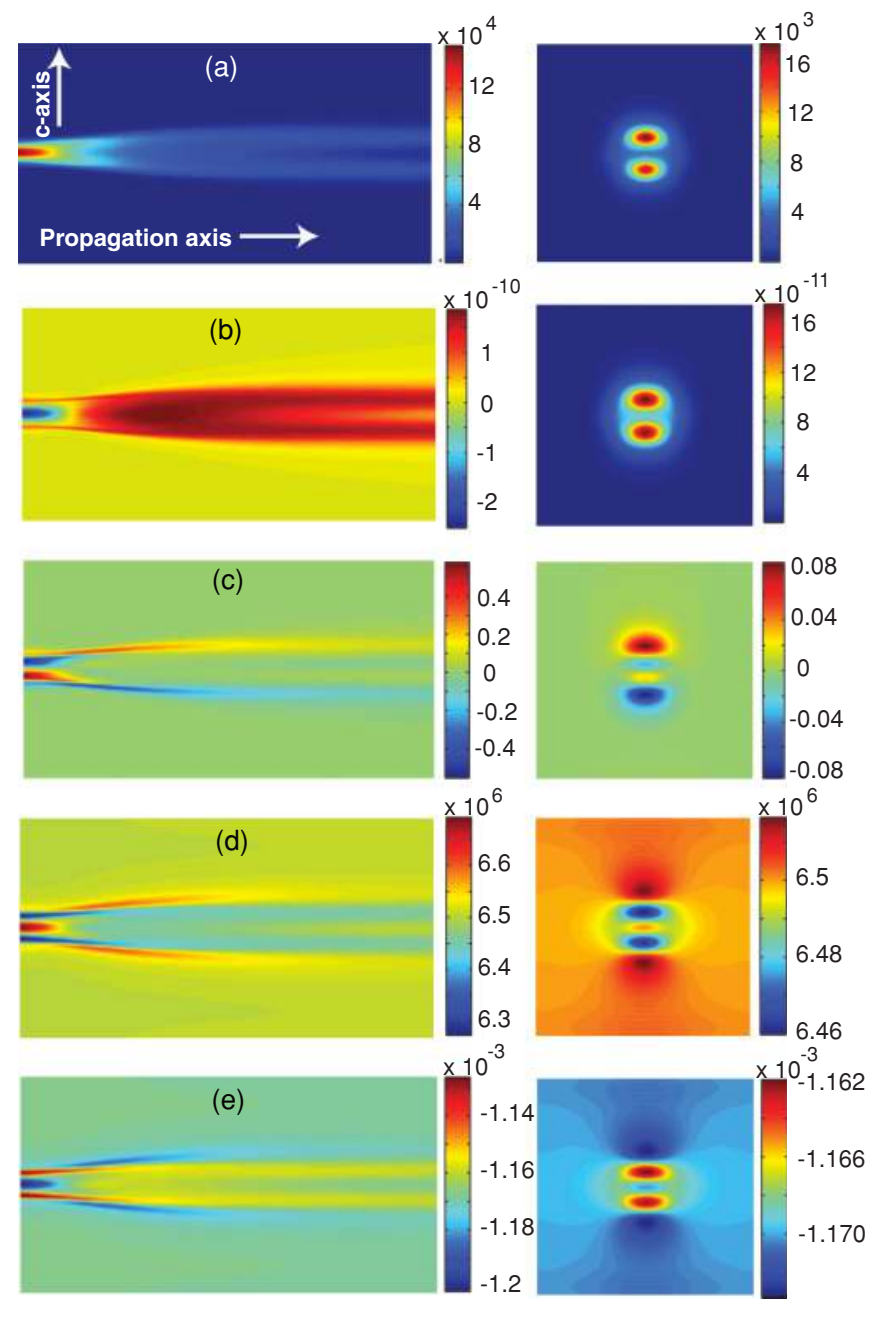

FIG. 4. (Color online) The PR process in an advanced state for a $140-\mu \mathrm{W}$ power beam $\left(I=1.5 \times 10^{5} \mathrm{~W} \mathrm{~m}^{-2}>I_{\text {th }}\right)$. Output transverse spatial distributions (left column) and spatial distribution along the propagation axis (right column) of beam intensity (a), total current component along the $c$ axis (b), space-charge density (c), total electric field component along the $c$ axis (d), and index modulation (e).
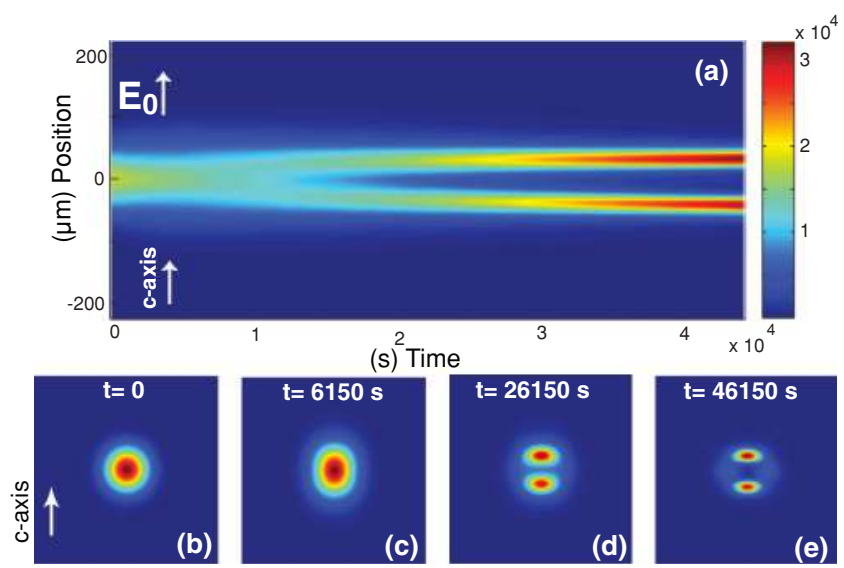

FIG. 5. (Color online) Splitting and self-focusing in two PR solitons of an intense $e$-polarized Gaussian beam. Output beam profile along the $c$ axis versus time (a), and pictures of the output beam at different times (b)-(e).

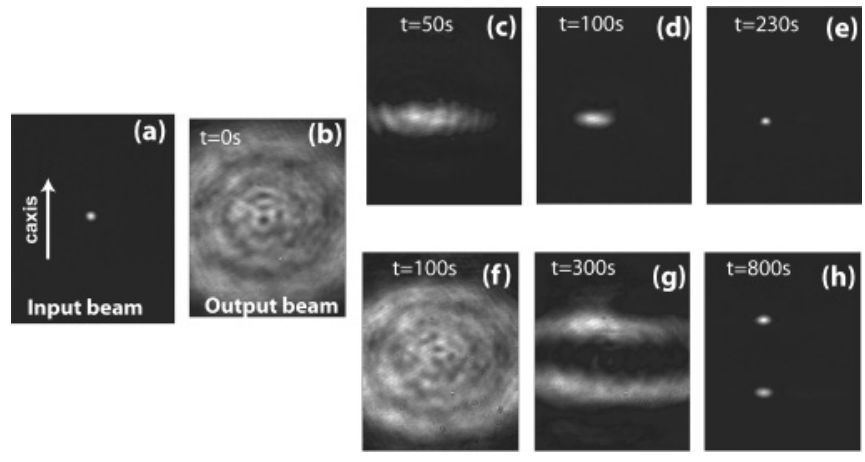

FIG. 6. Input (a) and output pictures of an $e$-polarized focused beam in linear regime (b) and the temporal dynamic of PR selffocusing (c)-(e) and splitting (f)- 0 (h) of the beam, respectively, for $45-\mu \mathrm{W}$ and $62-\mu \mathrm{W}$ beam power.

$\left(45 \mu \mathrm{W}: I \sim 1.4 \times 10^{5} \mathrm{~W} \mathrm{~m}^{-2}\right.$ ) where efficient beam selftrapping to a single spot is observed when the crystal is biased with $E_{0}=4 \times 10^{6} \mathrm{~V} \mathrm{~m}^{-1}$ as shown in Fig. 6(e). When beam power is increased up to $62 \mu \mathrm{W}\left(I \sim 2 \times 10^{5} \mathrm{~W} \mathrm{~m}^{-2}\right)$, splitting and self-focusing to two spots is now observed [Fig. 6(h)]. The corresponding temporal dynamics are given in both cases at three characteristic times [Figs. 6(c)-6(e) and Figs. 6(f) $-6(\mathrm{~h})$, respectively). In a first stage, for $62-\mu \mathrm{W}$ beam power, weak focusing of the output beam is observed [Fig. 6(f)]. Then, beam splits in two bright bow-shaped spots. For a longer exposure time, this bow-shaped self-focus gives rise to two PR spatial solitons [Fig. 6(h)]. Threshold intensity in this case is estimated to be approximately in the range $1.5-$ $2 \times 10^{5} \mathrm{~W} \mathrm{ym}^{-2}$. The intensity-dependent behavior observed experimentally is thus fairly well explained by our numerical model. Some mismatches are present between numerics and experiments which are mainly due to a poor knowledge of crystal parameters such as impurity concentrations. As a consequence, the characteristic times of PR effects, threshold intensity, or photovoltaic field amplitudes are difficult to determine accurately.

\section{CONCLUSION}

To conclude, we have presented a three-dimensional, timedependent numerical model that takes in account contributions of two photoactive centers (iron ions and polarons) that is successfully applied to explain PR beam self-focusing in biased $\mathrm{LiNbO}_{3}$. It reveals very different behaviors depending on the launched beam intensity. Numerical results explain, in fair agreement with the experiments, the prominent role played by the intensity-dependent photovoltaic field which compete with the applied field.

For a better physical insight into the processes, the phenomena involved in a simple case consisting of a homogeneously illuminated biased $\mathrm{LiNbO}_{3}$ are first studied. For low intensity, only iron centers play a role in the PR effect and the photovoltaic field is then constant. When beam intensity increases, polarons take into effect through an additional photovoltaic current. In this case, the photovoltaic current increases with intensity until all iron centers are ionized. It is demonstrated that for a given bias voltage, the total current can even change sign if the intensity is above or 
below a defined threshold intensity value. Moreover, analytical expressions of PV fields for iron ions and polarons, the total PV field with respect to light intensity and critical intensity, and perfectly matched numerical calculations are reported. The impact of this light-controlled current is then studied in the case of an illumination with a Gaussian beam. If input peak intensity is lower than the threshold intensity, beam self-trapping occurs, while for larger intensity, a more complex behavior is observed. Indeed, because of the intensity variation, both along the propagation axis and in the transverse plane, competition between drift and PV currents can either give the focusing or defocusing effect. As a consequence, the beam splits in the initial propagation distance to finally give multiple self-focused beams at a further distance. Finally, we report the corresponding experimental demonstration of beam splitting. Thanks to the presented 3D numerical model, comprehension of complex processes involved in PR experiments is accessible.
[1] P. Gunter and J. Huignard, Photorefractive Materials and their Applications III (Springer, Berlin, 2007).

[2] R. Gatass and E. Mazur, Nature Photonics 2, 219 (2008).

[3] M. Taya, M. Bashaw, M. Fejer, M. Segev, and G. Valley, Opt. Lett. 26, 1344 (2001).

[4] M. Taya, M. Bashaw, M. Fejer, M. Segev, and G. Valley, Opt. Lett. 21, 943 (1996).

[5] M. Chauvet, S. Chauvin, and H. Maillotte, Opt. Lett. 26, 1344 (2001).

[6] G. Couton, H. Maillotte, and M. Chauvet, J. Opt. B: Quantum Semiclass. Opt. 6, S223 (2004).

[7] E. Fazio, F. Renzi, R. Rinaldi, M. Bertolotti, M. Chauvet, W. Ramadan, A. Petris, and V. Vlad, Appl. Phys. Lett. 85, 2193 (2004).

[8] M. Segev, G. C. Valley, B. Crosignani, P. DiPorto, and A. Yariv, Phys. Rev. Lett. 73, 3211 (1994)

[9] A. A. Zozulya and D. Z. Anderson, Phys. Rev. A 51, 1520 (1995).

[10] P. Marquez-Aguilar, J. Sanchez-Mondragon, S. Stepanov, and V. Vysloukh, Phys. Rev. A 54, R2563 (1996).

[11] N. Fressengeas, J. Maufoy, and G. Kugel, Phys. Rev. E 54, 6866 (1996).
[12] B. Crosignani, P. DiPorto, A. Degasperis, M. Segev, and S. Trillo, J. Opt. Soc. Am. B 14, 3078 (1997).

[13] C. Denz, W. Królikowski, J. Petter, C. Weilnau, T. Tschudi, M. R. Belić, F. Kaiser, and A. Stepken, Phys. Rev. E 60, 6222 (1999).

[14] N. V. Kukhtarev, V. B. Markov, S. G. Odulov, M. S. Soskin, and V. L. Vinetskii, Ferroelectrics 22, 949 (1979).

[15] F. Jermann and J. Otten, J. Opt. Soc. Am. B 10, 2085 (1993)

[16] F. Jermann, M. Simon, and E. Kratzig, J. Opt. Soc. Am. B 12, 2066 (1995)

[17] F. Devaux, V. Coda, M. Chauvet, and R. Passier, J. Opt. Soc. Am. B 25, 1081 (2008).

[18] M. Chauvet, V. Coda, H. Maillotte, E. Fazio, and G. Salamo, Opt. Lett. 30, 1977 (2005).

[19] B. Sturman, M. Carrascosa, and F. Agullo-Lopez, Phys. Rev. B 78, 245114 (2008).

[20] M. Carrascosa, J. Villarroel, J. Carnicero, A. Garcia-Cabanes, and J. Cabrera, Opt. Express 16, 115 (2008).

[21] P. Herth, T. Granzow, D. Schaniel, T. Woike, M. Imlau, and E. Kratzig, Phys. Rev. Lett. 95, 067404 (2005). 